\title{
The optimization analysis on the vibration control of cylindrical shell with dynamic vibration absorber attached
}

\author{
Junjie Zhang \\ China Ship Development and Design Center, National Key Laboratory on Ship Vibration and Noise, \\ Wuhan, China \\ E-mail: potz@qq.com
}

Received 20 July 2018; accepted 5 August 2018

DOI https://doi.org/10.21595/vp.2018.20093

Check for updates

Copyright $(2018$ Junjie Zhang. This is an open access article distributed under the Creative Commons Attribution License, which permits unrestricted use, distribution, and reproduction in any medium, provided the original work is properly cited.

\begin{abstract}
The vibration control of cylindrical shell with dynamic vibration absorber (DVA) periodically and non-periodically attached is investigated. The coupled equations of cylindrical shell-DVA are established at first, and then the method to solve the equations is deduced. Parameters and coordinates of DVA are optimized to get the minimum mean square velocity (MSV) of cylindrical shell. Research results show that: (1) for DVA attached to cylindrical shell periodically, the number of DVA should be moderate, cannot be more or less; (2) the vibration reduction with DVA non-periodical arrangement attached to shell is better than that of periodical arrangement
\end{abstract}

Keywords: cylindrical shell, dynamic vibration absorber (DVA), mean square velocity (MSV), optimization algorithm, vibration reduction.

\section{Introduction}

DVA is applied to control the structure vibration and noise usually. In order to improve the control effect, parameters of DVA are optimized. The optimization of DVA in single degree-of-freedom (SDOF) and multi-degree-of-freedom (MDOF) system is classical optimization problems and has been already solved for a long time. For the continuous systems, the optimization of DVA published is focused on beam and plate, rarely about shell.

For a continuous structure such as a beam with DVA attached, vibration may be eliminated at the attachment point, while at other parts the vibration may be amplified. So, the vibration of whole structure may be more suitable to evaluate the vibration control effect of DVA. Lu [1] optimized the position and parameters of DVA to minimize the vibration of a simply supported beam. Nagaya [2] presented a method using neural network procedure to decrease vibration and noise from a plate. Wan [3] applied the admittance theory to control the vibration of a simply supported plate with DVA attached. Cheung [4] presented an optimization method to suppress vibration in plate, and got some interesting results. Fuller [5, 6] presented a technique to minimize the radiation sound of cylindrical shell. Achenbach [7] used the Lagrange equations and Donnell-Mushtari shell theory to suppress the vibration of cylindrical shell with DVA attached. It is showed that the control effect of DVA to radiation sound is good.

In the present paper, DVAs are applied to suppress vibration of cylindrical. DVAs are arranged in two categories: periodical and non- periodical arrangement. The PatternSearch optimization algorithm is used to minimize the MSV of shell. The results show that: (1) the number of DVA should be moderate, cannot be more or less for periodic DVAs arrangement; (2) the effect of vibration control with DVA non- periodical arrangement is better than the DVA with periodical arrangement. 


\section{Theory}

\subsection{Vibration of cylindrical shell}

Cylindrical shell with DVAs attached is showed in Fig. 1, the radius of shell is $R$, length is $L$. The axial, circumferential and radial coordinate are denoted by $x, \theta$ and $r$, respectively. $w, v$ and $u$ are the displacements in the $x, \theta$ and $r$ directions.

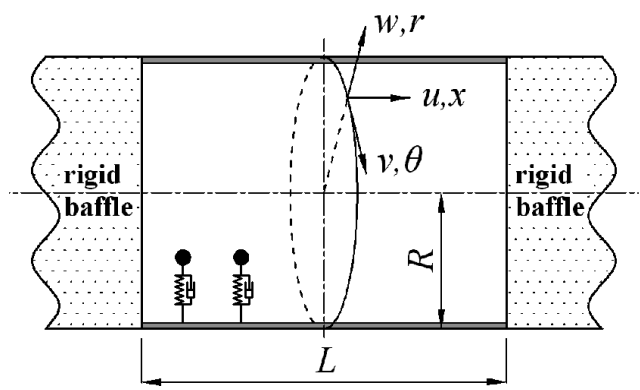

Fig. 1. Cylindrical shell attached with DVA

The vibration of cylindrical shell is described by Flügge equations. If the force is only excited in the radial direction, radial displacement of shell can be expressed according to the displacement impedance as:

$Z_{m n} W_{m n}^{\alpha}=F_{m n}^{\alpha}+T_{m n}^{\alpha}$

where, $W_{m n}^{\alpha}$ is radial displacement amplitude, $Z_{m n}$ is displacement impedance, $F_{m n}^{\alpha}$ is external force, $T_{m n}^{\alpha}$ is force exerted by DVA.

The radial displacement in time domain is expressed as:

$w=\sum_{\alpha=0}^{1} \sum_{m=1}^{\infty} \sum_{n=0}^{\infty} W_{m n}^{\alpha} \sin \left(n \theta+\frac{\alpha \pi}{2}\right) \sin \left(\frac{m \pi}{L} x\right)$.

\subsection{Force exerted to shell by DVA}

The sketch of DVA-shell is plotted in Fig. 2, the coupled equation is given by:

$m \ddot{z}_{0}+c\left(\dot{z}_{0}-\dot{w}_{0}\right)+K\left(z_{0}-w_{0}\right)=0$,

where, $m, c$ and $K$ is mass, damping coefficient and spring stiffness. $w_{0}$ is displacement of attachment point, $z_{0}$ is displacement of mass.

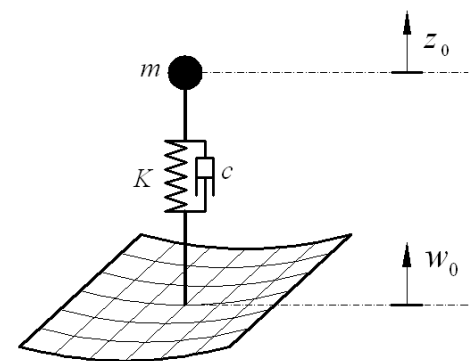

Fig. 2. The sketch of DVA-shell 
The force of DVA is:

$T\left(\theta_{0}, x\right)=c\left(\dot{z}_{0}-\dot{w}_{0}\right)+K\left(z_{0}-w_{0}\right)=T_{D V A} w_{0}$,

where, $T_{D V A}=\frac{m \omega^{2}(i c \omega+K)}{K-m \omega^{2}+i c \omega}$.

The orthogonality of trigonometric function is applied to Eq. (4), the force exerted to shell by the number $j$ DVA is:

$T_{m n_{j}}^{\alpha}=T_{j} t_{m n_{j}}^{\alpha} w_{j}$

where, $T_{j}=\frac{m_{j} \omega^{2}\left(i c_{j} \omega+K_{j}\right)}{K_{j}-m_{j} \omega^{2}+i c_{j} \omega}, w_{j}$ is attachment point of the number $j$ DVA and shell:

$t_{m n_{j}}^{\alpha}=\frac{\varepsilon_{n}}{\pi L R} \sin \left(n \theta_{j}+\frac{\alpha \pi}{2}\right) \sin \left(k_{m} x_{j}\right), \quad \varepsilon_{n}= \begin{cases}1, & n=0 \\ 2, & n \geq 1\end{cases}$

\subsection{The external force}

Using the orthogonality of trigonometric function, the external force of the number $i$ is:

$F_{m n_{-} i}^{\alpha}=F_{i} f_{m n_{-} i}^{\alpha}$,

where, $f_{m n_{i}}^{\alpha}=\frac{\varepsilon_{n}}{\pi L R} \sin \left(n \theta_{i}+\frac{\alpha \pi}{2}\right) \sin \left(k_{m} x_{i}\right)$.

\subsection{Solution and optimization}

\subsubsection{Vibration solution}

Substituting Eq. (5), (6) into Eq. (1), after rearranged, the expression can be written as:

$W_{m n}^{\alpha}=\frac{F_{m n}^{\alpha}+T_{m n}^{\alpha}}{Z_{m n}+L_{m n}}=\frac{\sum_{i} F_{i} f_{m n_{i}}^{\alpha}+\sum_{j} T_{j} t_{m n_{j}}^{\alpha} w_{j}}{Z_{m n}}$.

Substituting Eq. (7) into Eq. (2), the displacement of shell is expressed as:

$w(x, \theta)=\sum_{\alpha=0}^{1} \sum_{m=1}^{\infty} \sum_{n=0}^{\infty} \frac{\sum_{i} F_{0_{i}} f_{m n_{i}}^{\alpha}+\sum_{j} w_{j} T_{D V A_{j}} t_{m n_{D V} A_{j}}^{\alpha}}{Z_{m n}} \sin \left(n \theta+\frac{\alpha \pi}{2}\right) \sin \left(k_{m} x\right)$.

Set the $(x, \theta)$ to $\left(x_{j}, \theta_{j}\right)$ (attachment point of $j$ DVA and shell) each time, and solve the linear equations, the displacement $w_{j}$ is gotten. Then substituting $w_{j}$ into Eq. (7), the $W_{m n}^{\alpha}$ is solved.

The MSV is used to describe the vibration of the whole shell:

$\left\langle\dot{w} \dot{w}^{*}\right\rangle=\frac{1}{2 S} \int_{S}\left(\dot{w} \dot{w}^{*}\right) d S=\frac{1}{4} \sum_{\alpha=0}^{1} \sum_{m=1}^{\infty} \sum_{n=0}^{\infty} \frac{1}{\varepsilon_{n}} \dot{W}_{m n}^{\alpha} \dot{W}_{m n}^{\alpha *}$

\subsubsection{Optimization method}

The expression of MSV is complex in Eq. (9), so the minimum of MSV may be gained only by numerical optimization algorithm. The PatternSearch numerical optimization algorithm is recommended, and it can be called in Matlab. 
The flow diagram of optimization is showed in Fig. 3. The optimization algorithm is iterated until the difference between the two iteration MSV or step size reach to set value.

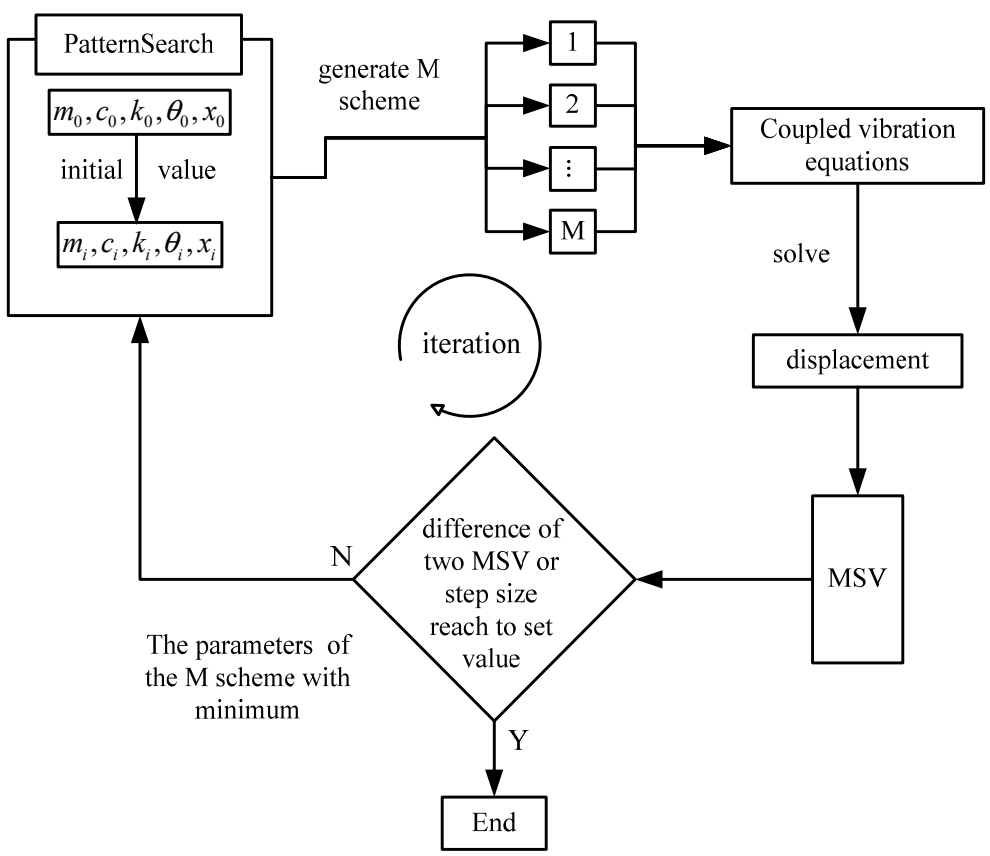

Fig. 3. The flow diagram of numerical optimization algorithm

\section{Numerical calculations and discussions}

Two unit forces are acted at position of $(0, L / 2)$ and $(\pi / 4,3 L / 4)$. Total mass of DVA is $10 \mathrm{~kg}$, damping coefficient is $c=200 \mathrm{~N} / \mathrm{m}$, frequency range is $50 \mathrm{~Hz}$ to $200 \mathrm{~Hz}$, and frequency interval is $1 \mathrm{~Hz}$. The participation modal of cylindrical shell $m=25, n=25$ (converged).

\subsection{Cylindrical shell without DVA attached}

Fig. 4 depicts the MSV of shell without DVA attached. There are two peaks in the curve, and the frequency is $121 \mathrm{~Hz}$ and $148 \mathrm{~Hz}$ respectively. The total MSV at frequency band [50-200 Hz] is $3.54 \mathrm{e}-07(\mathrm{~m} / \mathrm{s})^{2}$. In the following discussion, the value $3.54 \mathrm{e}-07(\mathrm{~m} / \mathrm{s})^{2}$ is used as the reference value to analyze the vibration reduction of cylindrical shell with DVA attached.

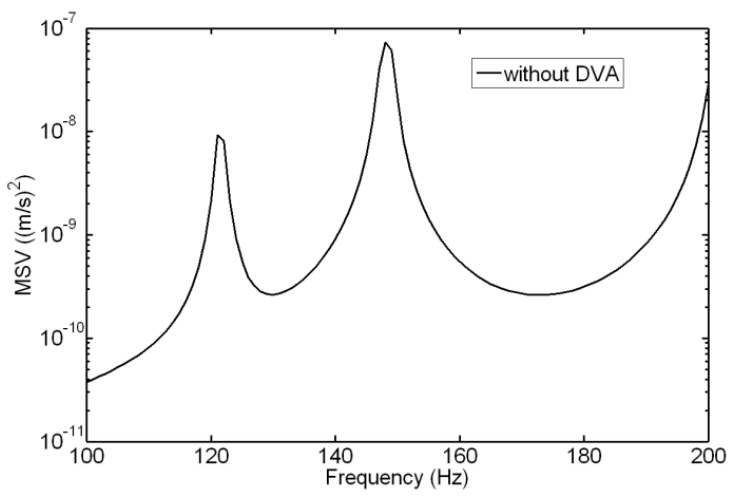

Fig. 4. MSV of shell without DVA attached 


\subsection{Cylindrical shell with DVA periodical arrangement}

The DVAs are arranged $6 \times 6$ on cylindrical shell periodically according to phononic crystals. The axial coordinates are $x=0.15 \mathrm{~m}, 0.45 \mathrm{~m}, 0.75 \mathrm{~m}, 1.05 \mathrm{~m}, 1.35 \mathrm{~m}, 1.65 \mathrm{~m}$; circumferential coordinates are $\theta=0, \pi / 3,2 \pi / 3, \pi, 4 \pi / 3,5 \pi / 3$. Mass of each DVA is $10 / 36=0.278 \mathrm{~kg}$.

\subsubsection{MSV with different spring stiffness}

The total MSV with different spring stiffness of DVA is analyzed, showed in Table1. The spring stiffness is equivalent to characteristic frequency by the formula $\sqrt{k / m} / 2 \pi$. It is showed that the optimization stiffness is $2.37 \mathrm{e} 5 \mathrm{~N} / \mathrm{m}$ (characteristic frequency $147 \mathrm{~Hz}$ ), and the total MSV is $1.56 \mathrm{e}-7(\mathrm{~m} / \mathrm{s})^{2}$. In the Table 1 , the vibration reduction is about $2.4 \mathrm{~dB}-3.6 \mathrm{~dB}$, so when the coordinates of DVAs and total mass are fixed, if only the spring stiffness is optimized, one cannot get the better vibration control effect.

Table 1. MSV with different spring stiffness

\begin{tabular}{|c|c|c|c|c|c|c|}
\hline Frequency $(\mathrm{Hz})$ & 50 & 100 & 121 & 148 & 200 & 147 (optimum) \\
\hline MSV $(\mathrm{m} / \mathrm{s})^{2}$ & $2.06 \mathrm{e}-7$ & $1.77 \mathrm{e}-7$ & $1.64 \mathrm{e}-7$ & $1.57 \mathrm{e}-7$ & $1.95 \mathrm{e}-7$ & $1.56 \mathrm{e}-7$ \\
\hline Vibration reduction $(\mathrm{dB})$ & 2.4 & 3.0 & 3.3 & 3.5 & 2.6 & 3.6 \\
\hline
\end{tabular}

\subsubsection{MSV with different total mass}

Keep the stiffness $2.37 \mathrm{e} 5 \mathrm{~N} / \mathrm{m}$ constant, and change the total mass of DVA to analyze the influence of the total mass to total MSV, expressed in Table 2. It is showed that the total MSV decreases as the total mass increase, but when the total mass excesses $15 \mathrm{~kg}$, the total MSV is no obvious decrease. So, it is not wise to get more vibration reduction through sustaining to increase the total mass.

Table 2. MSV with different total mass

\begin{tabular}{|c|c|c|c|c|c|c|}
\hline Total mass (kg) & 5 & 10 & 15 & 20 & 25 & 30 \\
\hline MSV (m/s) & $2.49 \mathrm{e}-7$ & $1.56 \mathrm{e}-7$ & $1.26 \mathrm{e}-7$ & $1.14 \mathrm{e}-7$ & $1.09 \mathrm{e}-7$ & $1.07 \mathrm{e}-7$ \\
\hline Vibration reduction(dB) & 1.5 & 3.6 & 4.5 & 4.9 & 5.1 & 5.2 \\
\hline
\end{tabular}

\subsubsection{MSV with spring stiffness and coordinates optimized for different DVAs arrangement}

Keep the total mass $10 \mathrm{~kg}$ constant. The periodic arrangement schemes of DVAs are $6 \times 6,5 \times 5$, $4 \times 4,3 \times 3,2 \times 2$, and the results of both stiffness and coordinates optimized are showed in the Table 3. Total MSV decreases at first, and then increases as the number of DVA decreases. So, for periodic DVA arrangement, the number of DVA should be moderate, cannot be more or less.

Table 3. Optimize spring stiffness and coordinates for different DVAs arrangement

\begin{tabular}{|c|c|c|c|}
\hline Scheme number & DVAs arrangement & Total MSV $(\mathrm{m} / \mathrm{s})^{2}$ & Vibration reduction(dB) \\
\hline 1 & $6 \times 6$ & $1.04 \mathrm{e}-7$ & 5.3 \\
\hline 2 & $5 \times 5$ & $8.14 \mathrm{e}-8$ & 6.4 \\
\hline 3 & $4 \times 4$ & $6.04 \mathrm{e}-8$ & 7.7 \\
\hline 4 & $3 \times 3$ & $7.16 \mathrm{e}-8$ & 6.9 \\
\hline 5 & $2 \times 2$ & $8.99 \mathrm{e}-8$ & 6.0 \\
\hline
\end{tabular}

For the $6 \times 6$ DVAs arrangement, if only the spring stiffness is optimized, the total MSV is 1.56e-7 (m/s) $)^{2}$ (showed in Table 1); if spring stiffness and coordinates both are optimized, the total MSV is $1.04 \mathrm{e}-7(\mathrm{~m} / \mathrm{s})^{2}$ (showed in Table 3 ). So, through multiple parameters optimization, the total MSV decreases more about 33.6\%. Compared to Table 3, it can be gotten less MSV through multiple parameters optimization relative to increase total mass. 


\subsection{Cylindrical shell with DVA non-periodical arrangement}

For the DVA non- periodical arrangement, mass of each DVA is equal and equal to $10 \mathrm{~kg} /$ number of DVA. Stiffness, circumferential and radial coordinates of each DVA are optimized. The total MSV with different number of DVA is showed in Table 4. With the increase of number of DVA, the total MSV of shell is decreased effectively, but when the number of DVA is greater than 4 , the MSV decrease is no obvious.

Table 4. The total MSV with different Scheme number

\begin{tabular}{|c|c|c|c|}
\hline Scheme number & Number of DVA & Total MSV $(\mathrm{m} / \mathrm{s})^{2}$ & Vibration reduction $(\mathrm{dB})$ \\
\hline Scheme 1 & 1 & $1.47 \mathrm{e}-07$ & 3.8 \\
\hline Scheme 2 & 2 & $6.56 \mathrm{e}-08$ & 7.3 \\
\hline Scheme 2 & 3 & $4.01 \mathrm{e}-08$ & 9.5 \\
\hline Scheme 4 & 4 & $2.82 \mathrm{e}-08$ & 11.0 \\
\hline Scheme 5 & 5 & $2.34 \mathrm{e}-08$ & 11.8 \\
\hline Scheme 6 & 7 & $2.20 \mathrm{e}-08$ & 12.1 \\
\hline
\end{tabular}

Comparing the Table 3 and Table 4, it is showed that when the number of DVA is equal or greater than 3 , the vibration reduction with DVA non- periodical arrangement is better than that of periodical arrangement.

\section{Conclusions}

1) The coupled equations of DVA-shell are established, and the optimization method of vibration reduction is recommended.

2) For DVAs attached to cylindrical shell periodically according to photonic crystals, the number of DVAs should be moderate, cannot be more or less.

3) The vibration reduction with DVA non-periodical arrangement attached to shell is better than that of periodical arrangement.

\section{References}

[1] Lu Yihua Research on Undamped Vibration Absorber Attached to the Continuous Systems. Nanjing University of Aeronautics and Astronautics, Nanjing, 2005.

[2] Nagya K., Li. L. Control of sound noise radiated from a plate using dynamic absorber under the optimization by neural network. Journal of Sound and Vibration, Vol. 208, Issue 2, 1997, p. 289-298.

[3] Wan Minhong The Study on Vibration Control of Thin Plate. Northwestern Polytechnical University, Xi'an, 2007.

[4] Cheung Y. L., Wong W. O. Ho and H2 optimizations of a dynamic vibration absorber for suppressing vibrations in plates. Journal of Sound and Vibration, Vol. 320, Issues 1-2, 2009, p. 29-42.

[5] Fuller C. R., Silcox R. J. Active structural acoustic control. Journal of Acoustical Society of America, Vol. 91, 1992, p. 519.

[6] Fuller C. R., Maillard J. P., Mercadal M., Von Flotow A. H. Control of aircraft interior noise using globally detuned vibration absorbers. Proceedings of 1st CEAS/AIAA Aeroacoustics Conference, Munich, 1995, p. 615-624.

[7] Achenhach J. D. Effect of a vibrating substructure on acoustic radiation from a cylindrical shell. Transactions of the ASME, Vol. 114, Issue 3, 1992, p. 312-318. 